\title{
Study on Feature Extraction of Semiconductor Gas Sensor Based on Prony's Method
}

\author{
Yoshinori Takei*, Shin Koyama, Hidehito Nanto and Takashi Mihara ${ }^{1}$ \\ Research Laboratory for Integrated Technological Systems, \\ Kanazawa Institute of Tech., 3-1 Yatsukaho, Hakusan, Ishikawa 924-0838, Japan \\ ${ }^{1}$ Future Creation Laboratory, Olympus Corporation, \\ 2-3 Kuboyama-cho, Hachioji-shi, Tokyo 192-8512, Japan \\ (Received February 7, 2009; accepted April 10, 2009)
}

Key words: semiconductor gas sensor, feature extraction, Prony's method

In this study, feature extraction from a transient response of a semiconductor gas sensor is investigated. For the sensor response given as the step response of the firstorder lag system, the sensor output can be approximated as a sum of exponentials by Prony's method. The model parameters can be estimated as the time constant and the gain, which are inherent parameters for each gas. The parameters can be used as features for the discrimination of gases. Using the extracted features, the discrimination of gases will be achieved even if only a single sensor is used. The parameter estimation procedure is basically an offline algorithm. We will then show an online estimation procedure for feature extraction with a low computational burden.

\section{Introduction}

Recently, sensing systems for gases or odorants, which are called electric nose systems, have attracted much attention for various areas, e.g., in food processing, medical and welfare areas. Semiconductor gas sensors are well known commercialized gas sensors for reasons of their temporal stability and other properties. In electric nose systems, the gas sensor as an element of the sensor array should basically have a high selectivity for sample discrimination. Then, the amplitude of the sensor output in the steady state can be used as a feature. Semiconductor gas sensors generally have a low selectivity; therefore, feature extraction in the transient state has been proposed to improve the discrimination by the augmentation of the feature vector. ${ }^{(1)}$

In this paper, we consider feature extraction from a transient response of a semiconductor gas sensor. For the sensor response given as the step response of the first-order lag system, ${ }^{(2)}$ the sensor output can be approximated as a sum of exponentials by Prony's method. ${ }^{(3)}$ The model parameters can be estimated as the time constant and gain, which are inherent parameters for each gas. The parameters can be used as features

"Corresponding author: e-mail: takei@neptune.kanazawa-it.ac.jp 
for the discrimination of gases by multivariate analysis. Using the extracted features, the discrimination of gases can be achieved even if only a single sensor is used. The parameter estimation procedure basically uses an offline algorithm, we will then show an on-line estimation procedure for feature extraction with a low computational burden.

\section{Experimental Methods}

Figure 1 shows the experimental setup of the gas sensor system. As a semiconductorbased gas sensor, TGS822 (Figaro Eng. Inc.) is selected. The sensor has a low selectivity, but this means that it can detect various gases; thus, it is suitable to study the relationship between the extracted features and characteristics of sample gases from the measurement data.

Sensor signals can be collected through a low pass filter and an amplifier circuit board using an AD converter with a laptop PC. The sensor is located at the bottom of a closed chamber $(5,000 \mathrm{ml})$. Sample gases are injected using a microsyringe from the top of the chamber, where the concentration of each sample gas is adjusted to $200 \mathrm{ppm}$. Then, the stepwise response to the injected gas is measured.

Figure 2 shows typical transient responses to sample gases. This shows that the characteristics of the sensor responses to the gases are different. Then, we can see that the transient response can provide information, which can be characterized by the steadystate value and time constant.

\section{Sensor Respose Modeling}

Assuming that the gas reaction on the surface of the sensor can be regarded as a firstorder reaction, the transient response obtained by the sensor can be modeled as the step response of the discrete first-order lag system. ${ }^{(1)}$

Then, we obtain

$$
y_{k}=C(1-\exp (-k \Delta t / \tau))
$$

where $\tau$ is a time constant and $C$ is a steady-state value that denotes the initial concentration or saturation mass of adsorption. Equation (1) shows a sensor response

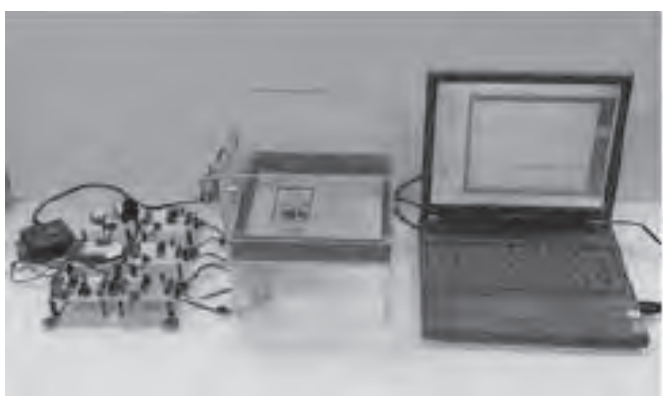

Fig. 1. Gas sensor system. 


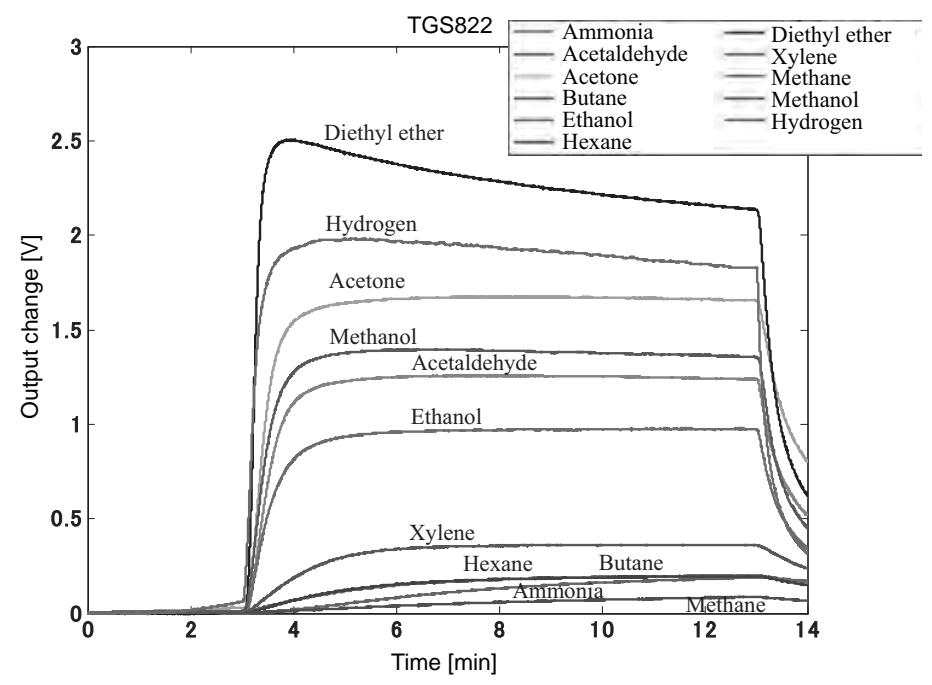

Fig. 2. Typical transient response to gases.

with a single gas injected stepwise. Therefore, it is important that these parameters are estimated to extract features of each gas. To estimate the parameters, we use Prony's $\operatorname{method}^{(4)}$ as follows.

Here, we review and consider Prony's method, which approximates the sensor response using a sum of exponentials as

$$
y_{k}=\sum_{i=1}^{n} m_{i} r_{i}^{k}
$$

where $r_{i}=\exp \left(-\Delta t / \tau_{i}\right)$ and $n$ is the order of the model.

The z-transform of eq. (2) can be obtained as

$$
Y(z)=\sum_{i=1}^{n} \frac{m_{i}}{1-r_{i} z^{-1}}=\frac{D(z)}{C(z)},
$$

where

$$
C(z)=1+\sum_{i=1}^{n} c_{i} z^{-i}, \quad D(z)=\sum_{i=0}^{n-1} d_{i} z^{-i}
$$

and $c_{i}$ and $d_{i}$ are real coefficients.

On the other hand, we can also approximate the sensor output using the autoregressive (AR) model represented by eq. (4). 


$$
y_{k}+\sum_{i=1}^{L} a_{i} y_{k-i}=e_{k}
$$

where $a_{i}$ is the AR coefficient and $e_{k}$ is the error. Then, the AR coefficients can be obtained by the least-squares (LS) method. Then, the z-transform of eq. (4) can be obtained as

$$
Y(z)=\frac{E(z)}{1+\sum_{i=1}^{L} a_{i} z^{-i}},
$$

where $E(z)$ corresponds to the z-transform of $e_{k}$. Comparing eqs. (3) and (4), it is clear that the coefficients in $C(z)$ are equivalent to the AR coefficients. Thus, the following characteristic equation is solved,

$$
1+\sum_{i=1}^{L} a_{i} x^{i}=0
$$

where $x^{i}=z^{-i}$, and $\tau_{i}$ can be obtained as follows using real roots of eq. (6).

$$
\tau_{i}=\Delta t / \log x_{i}
$$

The estimated time constant can be obtained as an inherent parameter for each gas, and denotes the index of the number of input gas components.

The steady-state value $m_{i}$ can also be estimated by minimizing the following error.

$$
E=\sum_{k=0}^{N}\left(y_{k}-\sum_{i=1}^{n} m_{i} r_{i}^{k}\right)^{2}
$$

where $N$ is the data length. As $\partial E / \partial m_{i}=0$, we then have the estimate of $m_{i}$;

$$
\boldsymbol{m}=\left(R_{N}^{T} R_{N}\right)^{-1} R_{N}^{T} \boldsymbol{y}_{N}
$$

where

$$
\begin{gathered}
\boldsymbol{m}=\left[m_{1}, m_{2}, \cdots, m_{n}\right]^{T}, \\
\boldsymbol{y}_{N}=\left[y_{0}, y_{1}, \cdots, y_{N}\right]^{T},
\end{gathered}
$$

and the Vandermonde matrix $R$ is given as 


$$
R_{N}=\left[\begin{array}{cccc}
1 & 1 & \cdots & 1 \\
r_{1}^{2} & r_{2}^{2} & \cdots & r_{n}^{2} \\
\vdots & \vdots & \ddots & \vdots \\
r_{1}^{N} & r_{2}^{N} & \cdots & r_{n}^{N}
\end{array}\right]
$$

Using the estimated $m_{i}$, the sensor output corresponding to the selected $\tau_{i}$ in mixed gas components can also be approximately calculated. Then, the extraction and selection of $\tau_{i}$ and the calculation of the corresponding $m_{i}$ can be used to modify the selectivity of the gas sensor.

In eq. (1), to remove the effect of the constant term as the offset, the sensor output data can be redefined and converted to the following differential data using the data at the $l$ step ahead ${ }^{(2)}$

$$
y_{k} \equiv y_{k+l}-y_{k},
$$

where $0 \leq k \leq N-l-1, l \geq 1$. Then, eq. (2) with a single gas can be represented as

$$
\begin{aligned}
y_{k} & =C_{i}\left(1-\exp \left(-l \Delta t / \tau_{i}\right)\right) \exp \left(-k \Delta t / \tau_{i}\right) \\
& =C_{l} \exp \left(-k \Delta t / \tau_{i}\right)
\end{aligned}
$$

where

$$
C_{l}=C_{i}\left(1-\exp \left(-l \Delta t / \tau_{i}\right)\right)
$$

Although the above algorithm is an offline algorithm, if the method can be extended to an on-line algorithm we can expect that the algorithm can be applied to various problems. Thus, we hereafter consider a recursive updating of the estimates of the parameters.

Equation (4) can be represented as follows:

$$
\begin{gathered}
y_{k}=\boldsymbol{a}_{L, k} \boldsymbol{y}_{L, k}+e_{k}, \\
\boldsymbol{a}_{L, k}=\left[a_{1}, a_{2}, \ldots, a_{L}\right]^{T}, \\
\boldsymbol{y}_{L, k}=-\left[y_{k-1}, y_{k-2}, \cdots, y_{k-L}\right] .
\end{gathered}
$$

Here, the AR coefficient vector $\boldsymbol{a}_{n, k}$ can be estimated by the recursive least-squares (RLS) method.

$$
\begin{gathered}
\hat{\boldsymbol{a}}_{L, k}=\hat{\boldsymbol{a}}_{L, k-1}+K_{k}\left(y_{k}-\hat{\boldsymbol{a}}_{L, k-1} \boldsymbol{y}_{L, k}\right), \\
K_{k}=\frac{\boldsymbol{p}_{k} \boldsymbol{y}_{L, k}^{T}}{R},
\end{gathered}
$$




$$
\boldsymbol{p}_{k}=\boldsymbol{p}_{k-1}-\frac{\boldsymbol{p}_{k-1} \boldsymbol{y}_{L, k}^{T} \boldsymbol{y}_{L, k} \boldsymbol{p}_{k-1}}{\left(R+\boldsymbol{y}_{L, k} \boldsymbol{p}_{k-1} \boldsymbol{y}_{L, k}^{T}\right)}
$$

where we set the parameters as $\hat{\boldsymbol{a}}_{L, 0}=0, R=20$ and $\boldsymbol{p}_{0}=2 \times 10^{3} I$ in the following simulation.

Considering a numerical aspect, the real roots of eq. (8) can be obtained by eigenvalue decomposition of the following $L$-th order companion matrix related to the time series data:

$$
\left[\begin{array}{c}
y_{k-L+1} \\
y_{k-L+2} \\
\vdots \\
y_{k-1} \\
y_{k}
\end{array}\right]=\left[\begin{array}{ccccc}
0 & 1 & 0 & \cdots & 0 \\
0 & 0 & 1 & \cdots & \\
\vdots & \vdots & \vdots & \ddots & \vdots \\
0 & \cdots & \cdots & 0 & 1 \\
-a_{L} & \cdots & \cdots & -a_{2} & -a_{1}
\end{array}\right]\left[\begin{array}{c}
y_{k-L} \\
y_{k-L+1} \\
\vdots \\
y_{k-2} \\
y_{k-1}
\end{array}\right]
$$

The steady-state value $m_{i}$ can also be estimated using eq. (9). The $R$ matrix shown in eq. (10), however, is required to reconstruct all the elements for each sampling time. The computational burden of this updating must be reduced.

The model parameters $\tau_{i}$ and $m_{i}$ are time invariant. Therefore, we set the $\boldsymbol{r}_{i}$ as the LS estimate at each sampling step without reconstructing all the elements. Then, we can use the following matrix instead of $R$ in eq. (10)

$$
\hat{R}_{N}=\left[\begin{array}{cccc}
1 & 1 & \ldots & 1 \\
r_{1,1} & r_{2,1} & \ldots & r_{n, 1} \\
\vdots & \vdots & \ddots & \vdots \\
r_{1, N}^{N} & r_{2, N}^{N} & \ldots & r_{n, N}^{N}
\end{array}\right]
$$

where $r_{i, k}$ denotes the estimate of $r_{i}$, obtained in the $k$ step. Then, $m_{i}$ can be given as

$$
\hat{\boldsymbol{m}}=\left(\hat{R}_{N}^{T} \hat{R}_{N}\right)^{-1} \hat{\boldsymbol{R}}_{N}^{T} \boldsymbol{y}_{N}
$$

Hereafter we assume that the sample gases are loaded as a unit gas. Then, the time constant excluding the noise term can be obtained using

$$
\tau_{k}=\max \left(\tau_{i}\right)
$$

where the subscript $k$ means that the parameter is obtained in the $k$ step, and then the $\mathrm{R}$ matrix is shrunk as

$$
\hat{\boldsymbol{r}}_{k}=\left[1, r_{1,1}, \ldots, r_{1, k}^{k}\right]^{T}
$$

Then, $m_{k}$ can easily be updated by 


$$
m_{k}=\frac{d_{k-1}+r_{1, k}^{k} y_{k}}{b_{k-1}+\left(r_{1, k}^{k}\right)^{2}}
$$

where

$$
b_{k}=\hat{\boldsymbol{r}}_{k}^{T} \hat{\boldsymbol{r}}_{k}, d_{k}=\hat{\boldsymbol{r}}_{k}^{T} \boldsymbol{y}_{k} .
$$

From eq. (3), $C$ can be obtained using

$$
C_{k}=m_{k} /\left(1-\exp \left(-l \Delta t / \tau_{k}\right)\right)
$$

Then, we have derived a simplified and recursive estimation algorithm for estimating the parameters from the sensor response to a unit gas.

\section{Results and Discussion}

In this section, we consider the application of the proposed method to the analysis of practical data. A single semiconductor-type sensor, TGS822, is used. The order of the AR model is $L=15$, and the difference interval $l=2,000$. Using the closed chamber shown in Fig. 1, the response of the sensor to each gas is measured. Six sample gases are prepared, which are shown in Figs. 5 and 6. The measurement time is $10 \mathrm{~min}$, which includes 30,000 data points. The sampling rate is $20 \mathrm{~ms}$. Figure 3 shows the typical response to ethanol gas. In Fig. 3, the solid line indicates the measurement sensor output. The dashed lines indicate the results of the conventional method based on the offline procedure $^{(2)}$ and those of the proposed method included in eq. (22), which was performed recursively. The conventional method is a batch process; that is, the entire data are used to estimate only one set of parameters. On the other hand, the proposed method provides

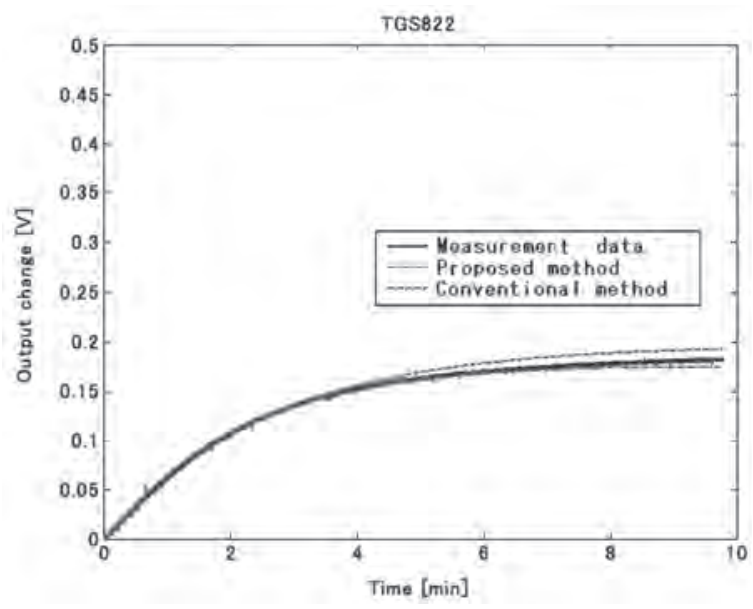

Fig. 3. Transient response to ethanol. 
an estimated sensor output at each step using limited data. Figures 3 and 4 show that the proposed method can be used to estimate the real response curve similarly to the conventional method.

Figures 5 and 6 show the convergence of the estimates when comparing the proposed method with the conventional method. They show that with increasing data, the estimates converge and the simplified gain estimation can be equivalent to that of the conventional method. Note that in Figs. 5 and 6, the time constant and saturation mass differ between gases and this shows that the parameters can be used to extract features of the gases.

Furthermore, regarding numerical aspects, the proposed method could process data obtained over $10 \mathrm{~min}$ within $0.837 \mathrm{~min}$. On the other hand, the conventional method,

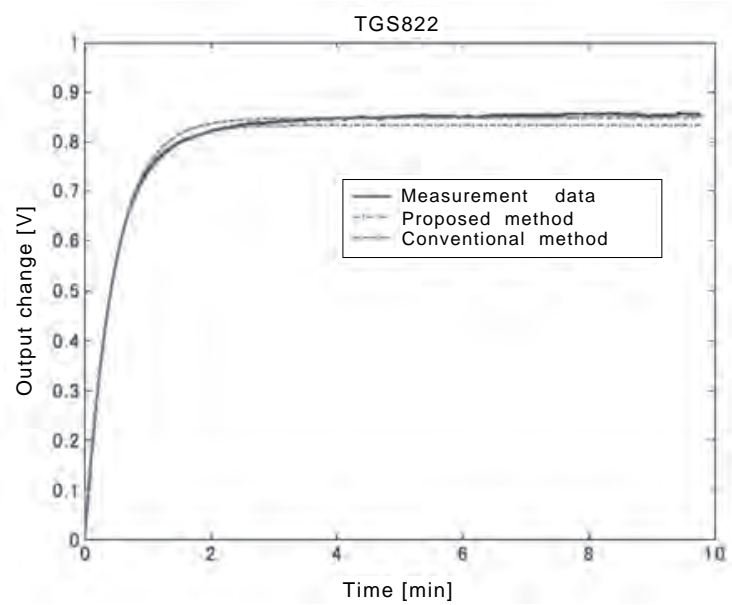

Fig. 4. Transient response to butane.

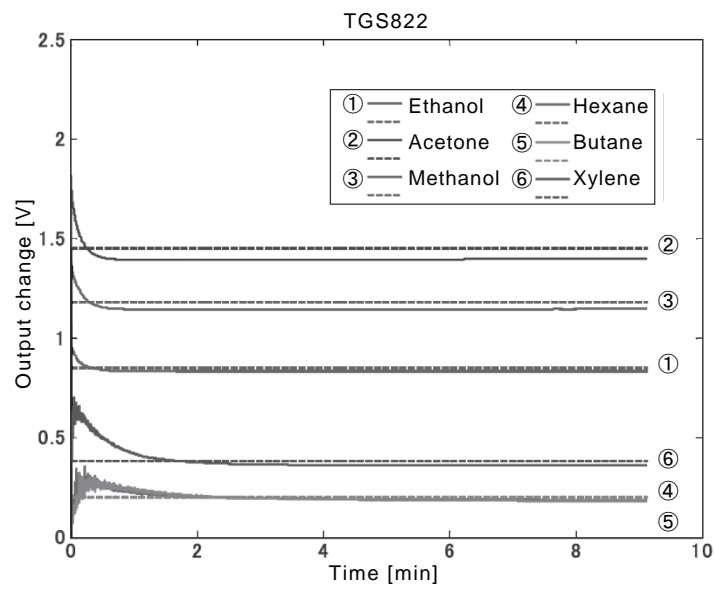

Fig. 5. Time constant for each gas in time elapsed. 


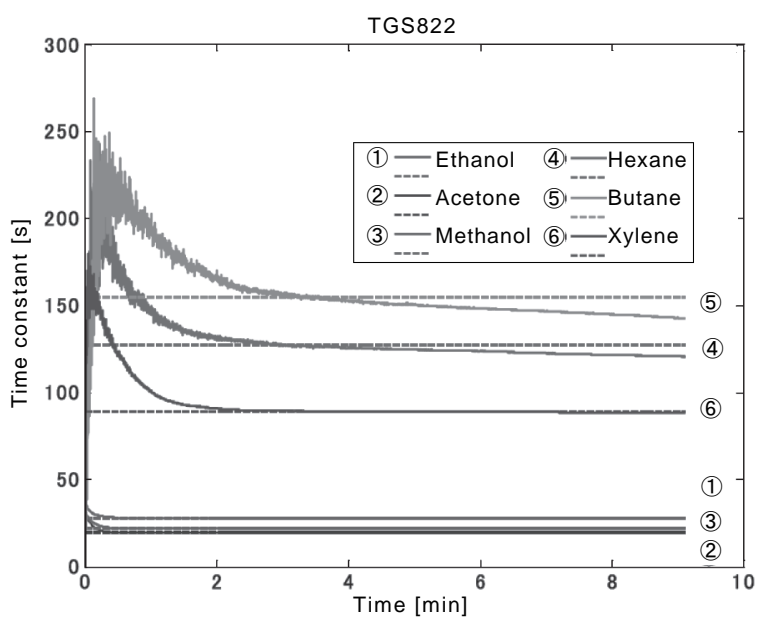

Fig. 6. Saturation mass for each gas in time elapsed.

which includes an RLS procedure for AR coefficients required 50 min under the same conditions with different calculations of the Vandermonde matrix corresponding to eqs. (10) and (18).

\section{Conclusions}

In this study, feature extractions from the transient response of a gas sensor were investigated. For the sensor response given as the step response of the first-order lag system, the sensor output has been approximated as a sum of exponentials by Prony's method. The model parameters of the time constant and gain, which are inherent parameters for each gas, can be used as features for discriminating gases. Furthermore, we showed that the on-line estimation procedure and the proposed algorithm can give equivalent results to the conventional algorithm with a low computational burden.

\section{Acknowledgements}

The authors wish to thank T. Asada for his excellent assistance with the experiments. This work was partly supported by the Ministry of Education, Science, Sports and Culture, Grant-in-Aid for No. 20760284, 2008.

\section{References}

1 S. Seto, H. Kawabe, L. Shi, Y. Shimomura, T. Oyabu and T. Katsube: Sensors and Materials 18 (2006) 1.

2 S. Seto, H. Kawabe, Y. Shimomura, L. Shi, T. Katsube and T. Oyabu: IEEJ Trans. Sensors and Micromachines 125 (2005) 129 (in Japanese).

3 M. Nakamura, I. Sugimoto and H. Kuwano: Trans. SICE 31 (1995) 437.

4 Z. Iwai, I. Mizumoto, M. Nagata, M. Kumon and Y. Kubo: Trans. JSME C 71 (2005) 589. 